\title{
El aprendizaje del ritmo musical
}

\section{Claire Gérard}

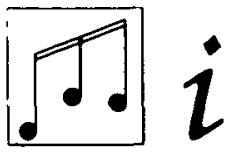

¿En qué actividades y soportes instrumentales se debe apoyar la educación del ritmo musical en cada edad? ¿Qué errores son "buenos» $y$ cuáles son "malos» en la organización rítmica del niño? ¿Hasta qué punto se separa la educación rítmica de una evolución espontänea? En este trabajo se verifican las respuestas a éstas y otras preguntas, lo que permite ir algo más lejos de la pedagogía del ritmo de los llamados «métodos activos»

En el panorama general de los trabajos de psicología experimental dedicados a la música, el ritmo aparece como el pariente pobre de la familia: aunque no está ausente de las grandes síntesis que se han esbozado, Dowling y Harwood (1986) o Sloboda (1985), Zenatti (1981) o Hargreaves (1986), los capítulos a él dedicados son los más breves. Por el contrario, en los métodos de iniciación musical llamados "métodos activos", desarrollados por diversos pedagogos sobre todo a partir de Orff, el ritmo ocupa el lugar de honor. Nuestro trabajo intenta llenar esta laguna en el conocimiento de los psicólogos y también plantear unas "propuestas» pedagógicas para comprobar experimentalmente, en unas determinadas situaciones, su eficacia. La enseñanza musical, sea cual sea el método utilizado, debe adaptarse a las aptitudes de los niños a los que va dirigida. Por ello, vamos a revisar brevemente las grandes líneas del desarrollo musical espontáneo de los niños, antes de centrarnos específicamente en el ritmo y en los problemas planteados por su aprendizaje.

Las investigaciones evolutivas demuestran que los cantos aparecen espontáneamente hacia los 12 meses según Dowling y Harwood (1986) y Kelley y Sotton-Smith (1987), y hacia los 18 meses según Sloboda (1985). Según estos mismos autores, hacia los 2-3 años los cantos creados por los niños son más extensos y comienzan a presentar signos de organización interna. A esta edad, los niños también son capaces de imitar fragmentos de las canciones que oyen, repitiendo las palabras más destacadas (Sloboda, 1985). Según Davidson, Mc Kernon y Gardner (1981) hacia los 3 años los niños empiezan a tener en cuenta el ritmo y la altura tonal, aunque Moog 
(1976) afirma que esta capacidad no es tan precoz (5 años). En cualquier caso, entre los 2-3 años la capacidad de imitación va a mejorar de tal forma que los niños serán capaces de reproducir canciones enteras. Los autores anteriormente citados afirman que la capacidad de reproducir el ritmo y el contorno musical aparecen antes que la capacidad de reproducir los intervalos exactos y la tonalidad dominante. Hacia los 5 años Sloboda (1985) considera que los niños reproducen perfectamente canciones familiares pero también disminuye su creatividad: a los niños les gusta repetir exactamente lo que han oído y están muy atentos a los detalles. Hacia los 6 años se produce una modificación importante: son capaces de mantener una tonalidad estable a lo largo de una canción, empezando y terminando sobre la tónica, incluso aunque determinados sonidos sean erróneos (Davidson, Mc Kernon y Gardner, 1981). A esta edad, los niños organizan también sus producciones en torno a un bloque de pulsación subyacente, mientras que a los 4 años esta organización regular sólo es transitoria, basada más en las palabras que en el ritmo musical propiamente dicho. Así, en las canciones, el ritmo a veces está basado en una organización verbal o en un contorno melódico. ¿Qué ocurre entonces con los ritmos que se presentan solos?

La capacidad de discriminación aparece muy pronto. Demany, Mc Kensie y Vurpillot (1977) demuestran que lactantes de 2-3 meses son capaces de distinguir dos estructuras rítmicas sencillas. Wasburn y Cohen (1984) confirman estos resultados, que implican que la discriminación se basa en la duración y el orden de los intervalos entre pulsaciones. A los tres meses, el niño puede distinguir dos estructuras, si en una de ellas se da un cambio de longitud de un intervalo. Desde el momento en que el niño es capaz de controlar sus movimientos, la reproducción de ritmos sencillos va a aportar una información diferente en relación con las tareas de discriminación. Como señala Fraisse (1956), sólo ella permite estudiar objetivamente las leyes de estructuración de un determinado ritmo. El análisis del rendimiento de los niños pone en evidencia que se da una clara evolución entre los 6 y los 9-10 años, pero es difícil precisar la naturaleza de esta evolución. En efecto, los autores (Stambak, 1960; Fraisse, 1974; Zenatti, 1981), que frecuentemente miden este rendimiento en función de los elementos recordados, no se ponen de acuerdo sobre la tasa óptima de estructuras rítmicas que un niño de 6, 8 ó 10 años puede reproducir correctamente, y tampoco precisan el tempo ni el lugar de los acentos en las estructuras temporales que presentan. Ahora bien, el ritmo musical, sobre todo el más sencillo, es una combinación de tres características: la rapidez o tempo, la organización temporal de los intervalos y la periodicidad de los acentos u organización intensiva. Por tanto, nos parece que está justificado el análisis de los ritmos reproducidos desde el punto de vista de estas tres características estructurales, en lugar de centrarnos en el número de elementos, que hacen olvidar la estructura. No obstante, tendremos en cuenta algunos de los resultados relacionados con variaciones en las modalidades de presentación de los modelos. Petzold (1966, en Zenatti, 1981) proponía tres series de pruebas: Presentación y reproducción de sonidos marcados con golpe, presentación al piano y reproducción con golpes, presentación al piano y respuesta cantada correspondiente al ritmo y la melodía. No se comprobó ninguna diferencia significativa entre las tres series de pruebas en los niños de la escuela primaria. Por el contrario, Zenatti (1981) obtuvo resultados sig- 
nificativamente mejores con una presentación mediante golpes, más que melódica, para la reproducción de estructuras sencillas, con niños de entre 5 años y 3 meses y 5 años y 8 meses. En otros trabajos se han utilizado formas de representación simbólica del ritmo, en las que un modelo visual servía de base a la reproducción. Stambak (1960) demostró que la comprensión de esta tarea sufría una evolución entre los 6 y los 8 años, pasando de un $64 \%$ a un $100 \%$, de la misma forma que el porcentaje de reproducciones correctas pasaba a un $77 \%$ entre los 6 y los 10 .

Para los pedagogos el ritmo es el elemento esencial para la sensibilización del niño en el terreno musical, especialmente en los métodos de Orff, Martenot, Willens y Kodaly. En estos métodos, la actividad tiene prioridad sobre el aprendizaje de la lectura o la escritura musical. Lo esencial, dicen Orff y Keetman (1967) consiste en seguir el mismo camino que el utilizado en el aprendizaje de la lengua materna: los niños "primero la hablan, luego la leen y escriben".

En todos estos métodos pedagógicos se multiplican las formas de presentación del ritmo. En los ejercicios de Martenot (1970, 1981a y b), Willems (1983), Kodaly (en Mialaret, 1979) se aconseja la utilización de un soporte verbal. El "hablar ritmado" acompasa el ritmo, facilitando su memorización. En este principio se basan las cantinelas. La pronunciación de los sonidos muestra la estructuración temporal e intensiva de la fórmula rítmica. En el método Kodaly, tal como lo describe Mialaret (1979), se utilizan onomatopeyas para facilitar el paso a la lectura rítmica. Por su parte, Willems defiende la utilización de pequeños diálogos que marquen el ritmo. En esta línea, Martelot propone los "lala..." o, incluso, la pronunciación del nombre de las figuras de las notas (“corchea, negra, blanca, fusa...»), preservando su duración. Los índices motores ocupan también un lugar importante en los ejercicios de reproducción del ritmo. Por ejemplo, Orff, como Wilens, propone acompañar el ritmo con paseos, carreras, balanceos.

Si no se puede poner en duda que incluir tal o cual índice puede, puntualmente, mejorar la percepción y reproducción de un determinado ritmo, el problema que se plantea, desde el punto de vista del psicólogo, es extraer, de entre los múltiples ejemplos presentados por los manuales pedagógicos, algunos principios generales y comprobar experimentalmente la validez de estos índices en las distintas etapas del desarrollo del niño. La investigación que vamos a presentar a continuación tenía como objetivo hacer un estudio de este tipo con niños de 6 años y medio a 8 años y medio, edad en la que empieza a sistematizarse la enseñanza de la música en la escuela primaria.

En la investigación que vamos a presentar se analizó la incidencia de distintas formas de presentación de secuencias rítmicas sobre la reproducción de estas secuencias por los niños. Se establecieron cuatro condiciones de presentación. En las cuatro condiciones los niños oían una secuencia rítmica de golpes de baqueta sobre una tabla de madera. En dos de estas condiciones auditivas, en las que no veian los gestos del experimentador, los niños oyen, además, onomatopeyas producidas por el experimentador (lalala" o "titita»), que acompañan a cada golpe. En las otras dos condiciones los niños oían la secuencia, pero además veían los gestos del experimentador, que estaba callado y daba golpes bien sobre un mismo emplazamiento 
o sobre un esquema espacial que representa el ritmo. En esta última situación, además el niño disponía de dicho esquema espacial para hacer su reproducción. Nuestra hipótesis suponía la existencia de una interacción entre la edad de los niños y la eficacia de los índices auditivos y/o visuales. En efecto, se puede suponer que para los niños más pequeños el relacionar informaciones auditivas y visuales sería una dificultad suplementaria, pues todavía establecen mal la correspondencia de unos índices aislados de dos canales sensoriales. Su atención se centraría en un solo canal, en este caso el auditivo, ya que la tarea que se les pedía era que reprodujeran el ritmo que ellos habían percibido. Para ellos la ayuda sólo podía proceder de los índices de diferenciación auditiva. Sólo los "grandes» (no habíamos preestablecido una edad exacta) podrían extraer ayuda de las informaciones visuales, al margen de la reproducción, y de la espacialización de las duraciones, lo que permitiría mejorar notablemente las reproducciones.

No utilizamos las formas de puntuación de respuestas habituales, que, en nuestra opinión, no reflejan los aspectos cualitativos de la reproducción. Evaluamos las reproducciones según 4 criterios, puntuando cada uno con 1 punto: el primer criterio permitiría diferenciar respuestas rítmicas de respuestas organizadas, el segundo valoraría la fidelidad de los intervalos reproducidos en relación con el modelo, el tercero se referiría al respeto de los acentos y el cuarto al respeto del tempo en la secuencia rítmica. Este sistema de anotación permitiría el análisis «global» de las escalas de reproducción (con 4 puntos) mediante un análisis más cualitativo de la frecuencia de aparición de distintos patrones de respuesta. En efecto, lo que nos interesa es la estructuración de las reproducciones, más que su fidelidad.

\section{METODO}

\section{Modelos rítmicos}

Se eligieron cuatro secuencias rítmicas para determinar los modelos (Codificados de M1 a M4) que los sujetos deberían reproducir. La Figura 1 presenta estos modelos, su tempo y el número de sus elementos, su división en medidas y el lugar de los acentos.

\section{Condiciones de presentación}

Se definieron 4 condiciones de presentación, codificadas como C. En $\mathrm{C} 1$ y $\mathrm{C} 2$, denominadas condiciones "auditivas", los sujetos no veían las manos del experimentador, pues las ocultaba una pantalla de cartón blanco de $30 \mathrm{~cm}$ de altura. En la condición C1, el experimentador golpeaba las secuencias rítmicas con una baqueta sobre una tabla de madera acompañando cada golpe con el sonido «La». En la condición C2, los golpes iban acompañados de onomatopeyas (que se tomaron prestadas de los talleres musicales) del tipo de "titita", en la que el sonido "ti" representaba duración breve y el sonido «ta» duración larga. Las condiciones C3 y C4 eran las llamadas «condiciones visuales». En C3 se suprimía la pantalla, y los sujetos veían al experimentador realizar la secuencia rítmica sin ninguna verbalización: se colocaba sobre la mesa una hoja de cartón blanco con un cuadrado negro de 15 por $15 \mathrm{~cm}$ en el centro, el experimentador golpeaba en 
el centro del cuadrado. En C4 también se colocaban sobre la mesa dos hojas de cartón blanco que representaban una espacialización de la sucesión de los intervalos temporales, de forma específica para cada secuencia: unos cuadrados negros (de $3,5 \mathrm{~cm}$ de lado) y unos rectángulos negros (de $3,5 \times 7 \mathrm{~cm}$ ) que representaban, respectivamente, los intervalos cortos y largos entre los golpes, se disponía en semicírculo, y el experimentador golpeaba sucesivamente de izquierda a derecha sobre estas figuras para realizar la secuencia rítmica. Los sujetos también tenian a mano hojas iguales para la reproducción de los modelos. La Figura 1 representa los códigos auditivos y visuales utilizados en $\mathrm{C} 2$ y $\mathrm{C} 4$, con cada uno de los 4 modelos.

\section{Sujetos}

El experimento se llevó a cabo en una escuela primaria de Antony (Hauts-de-Seine) con niños de preparatorio, $10^{\circ}$ y $2 .^{\circ}$ elemental. Todos los niños oían normalmente. Los «malos alumnos» (según los criterios de su profesora) fueron excluidos para evitar que se plantearan problemas auditivos y/o de atención. La experiencia se llevó a cabo con noventa y seis sujetos repartidos en tres grupos de 32 alumnos, cuya media de edad era 6 años y medio (de 6 años a 6 años y 9 meses), 7 años y medio (de 7 años a 7 años y 9 meses) y 8 años y medio (de 8 años a 8 años y 9 meses).

\section{Procedimiento y plan experimental}

Cada niño era examinado individualmente en una salita tranquila de la escuela. Los modelos presentados y las reproducciones de los niños se registraban en un magnetofón Sony TC-D5M para valorar posteriormente las reproducciones, pero también para controlar la estabilidad del modelo ejecutado ante el niño por el experimentador. El niño recibía la consigna de escuchar atentamente «la musiquilla» para hacer luego exactamente lo

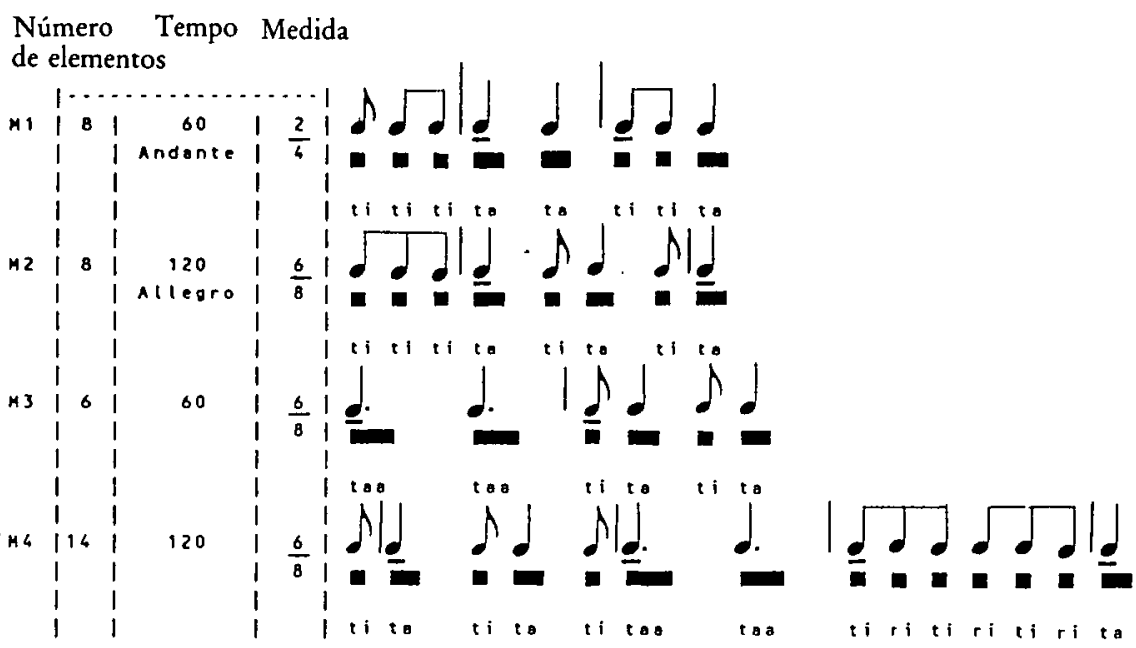


mismo. El resto de la consigna se adaptaba a las condiciones de presentación. Con cada modelo se llevaron a cabo dos ensayos sucesivos, alternando presentación y reproducción. Un ensayo consistía en la presentación de un modelo dos veces seguidas, separando las 2 presentaciones por la frase "otra vez». A su vez el niño debía reproducir dos veces el modelo y si lo deseaba podía repetir las onomatopeyas. Se valoraba la mejor de las dos reproducciones de cada ensayo. De forma sistemática la primera reproducción de cada ensayo resultó ser la mejor, por tanto era la que se tenía en cuenta.

Cada niño pasaba las 4 condiciones, de $\mathrm{C} 1$ a 4 , siguiendo órdenes contrabalanceados para comenzar tanto por condiciones auditivas como visuales. Dentro de cada tipo (auditivo o visual), siempre la situación más pobre en índices ( $\mathrm{C} 1$ o $\mathrm{C} 3$ ) era aquella con la que se comenzaba el experimento. Cada niño reproducía un modelo diferente por condición, por tanto se enfrentaba a los cuatro modelos seleccionados. Para que cada modelo también se pudiera presentar en todas las condiciones al conjunto de los sujetos, se preparó un plano, en cuadrado latino, de asignación de los modelos a las distintas condiciones. Este plano, añadido al contrabalanceado del orden, contribuyó a definir 8 subgrupos de cuatro sujetos por edad. Todos los factores se entrecruzaron de dos en dos, pero el plano del experimento no se pudo definir completamente (en una sola vez) en razón del cuadrado latino utilizado. Siendo A la edad, C las condiciones de presentación, M los modelos y $\mathrm{E}$ los ensayos, podemos escribir:

$$
\mathrm{S} 32<\mathrm{A} 3>\cdot \mathrm{C} 4 \cdot \mathrm{E} 2 \text { y } \mathrm{S} 32<\mathrm{A} 3>\cdot \mathrm{M} 4 \cdot \mathrm{E} 2
$$

\section{Puntuación de las reproducciones}

El análisis de los registros se llevó a cabo por dos «jueces». Cada reproducción se codificaba según un modelo de respuesta, en sucesión de 0 a 1 en cuatro columnas correspondiendo cada una a un aspecto de la reproducción. La primera columna correspondía a la "forma» de la reproducción, que si era poco pregnante se puntuaba 0 y si estaba bien estructurada en dos duraciones, una corta y otra más o menos larga, se puntuaba 1. La forma correspondiente a la nota 1 podría ser totalmente diferente a la del modelo. La segunda columna permitía decidir en qué casos la nota 0 significaría que una forma (pregnante o no) no respetaba la sucesión de intervalos cortos y largos del modelo, mientras que la nota 1 precisaría que al menos los 5 primeros golpes estaban organizados conforme al modelo. La tercera columna indicaba si se habían reproducido los acentos (conforme o no al modelo). En efecto, un ritmo es una organización intensiva además de temporal, y en el modelo figuraban los acentos: la nota cero indicaría que no se había descubierto ninguna variación de intensidad, la nota 1 indicaría que se había marcado una acentuación en determinados golpes en relación con otros. Por último, la $4 .^{2}$ columna se refería al tempo de las reproducciones: la nota 0 representaría una diferencia notable entre la rapidez del modelo y la de la reproducción, la nota 1 indicaría que el tempo se había respetado al menos en un $10 \%$.

A partir de este sistema de puntuación, sumando los puntos se podría obtener una nota total sobre 4 puntos, que permitiría un estudio global de la importancia de los diferentes factores del experimento: esto constituiría 
la primera parte del análisis de los resultados. También se podría analizar la frecuencia de la aparición de los distintos modelos para estudiar el tipo de organización de las reproducciones, lo que constituiría la segunda parte del análisis de los resultados.

\section{RESULTADOS}

\section{Estudio global}

Las notas totales obtenidas sobre 4 puntos por edad, condiciones de presentación y modelo figuran en el Cuadro 1 . El análisis de varianza realizado según el programa VAR3, permite demostrar que las reproducciones mejoran al aumentar la edad del niño $(F(2,84)=14,40 ; p<001)$. Las comparaciones parciales permiten precisar que los progresos registrados entre los 6 años y medio y los 7 y medio no son significativos (se pasa de una media de 1,76 con $\sigma 1,41$ a una media de 2,03 , con $\sigma 1,38$ ), mientras que la mejora entre los 7 años y medio y los 8 y medio es significativa ( $F$ $(1,56)=27,40 ; p<001)$. En esta edad no sólo mejoran las medias (pasando de 2,03 a 2,80 ), sino que también disminuye la variabilidad interindividual (pasando de $\sigma=1,38$ a $\sigma=1,21$ ), con lo que se franquea el "cabo" de la significación estadística. Señalaremos, de paso, que también en otros estudios con niños que hemos realizado aparece el mismo fenómeno: entre los 6 y los 9 años la mejora de las puntuaciones medias es paralela a la reducción de las desviaciones típicas, y las convergencias de estas dos evoluciones es la que marca básicamente el paso de los 7 a los 8 años.

Las condiciones de preentación muestran también un efecto global significativo ( $F$ " $(3,588)=3,26 ; p<0,25)$, pero, en cuanto a este factor, lo

\section{FIGURA 2}

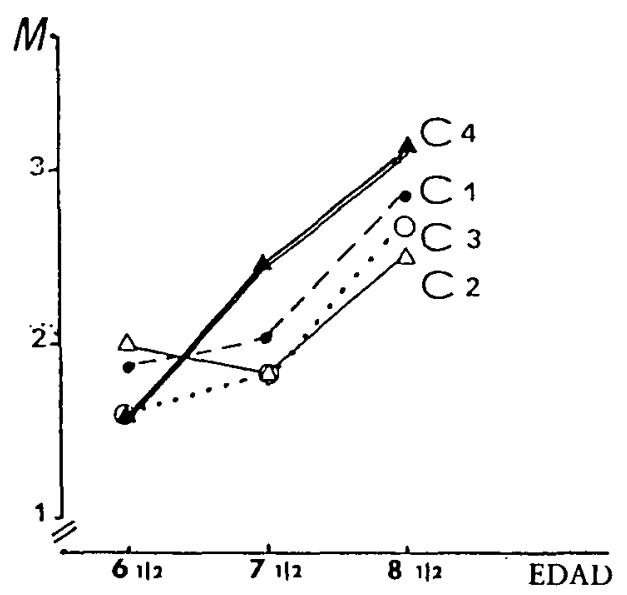

Evolución de las puntuaciones medias con la edad en cada condición.

C1: modelos acompañados de "la la la"

C2: modelos marcados por ati ti ta"

C3: observación de los gestos del examinador

C4: espacialización de las duraciones 
TABLA I

Puntuaciones medias en función de las condiciones de presentación $C$ para los 4 modelos ritmicos $M 1$ a $M 4$

\begin{tabular}{lcccccc}
\hline & & M1 & M2 & M3 & M4 & m \\
\hline 6 años 1/2 & C1 & 1,69 & 3,06 & 1,94 & 0,87 & 1,89 \\
& C2 & 1,62 & 2,87 & 2,94 & 0,44 & 1,97 \\
& C3 & 1,06 & 2,00 & 2,19 & 1,19 & 1,61 \\
& C4 & 1,69 & 1,69 & 1,69 & 1,25 & 1,58 \\
\cline { 3 - 7 } & m & 1,51 & 2,40 & 2,18 & 0,94 & 1,76 \\
\hline 7 años 1/2 & C1 & 1,87 & 2,94 & 2,25 & 1,19 & 2,06 \\
& C2 & 1,12 & 2,37 & 2,31 & 1,37 & 1,80 \\
& C3 & 1,25 & 2,87 & 2,06 & 1,06 & 1,81 \\
& C4 & 3,25 & 3,25 & 2,69 & 0,69 & 2,45 \\
\hline \multirow{2}{*}{8 años 1/2 } & m & 1,87 & 2,86 & 2,33 & 1,07 & 2,03 \\
& C1 & 3,19 & 3,31 & 2,56 & 2,31 & 2,84 \\
& C2 & 2,05 & 3,19 & 3,44 & 1,50 & 2,55 \\
& C3 & 2,31 & 3,56 & 3,12 & 1,81 & 2,70 \\
& C4 & 3,44 & 3,62 & 3,06 & 2,31 & 3,12 \\
\hline & m & 2,75 & 3,45 & 3,05 & 2,00 & 2,80 \\
\hline
\end{tabular}

C1: modelos acompañados de «la la la».

C2: modelos marcados por ati ti ta".

C3: observación de los gestos del examinador.

C4: espacialización de las duraciones.

que nos interesa es comprobar nuestra hipótesis, es decir, que se da una interacción entre la edad y las condiciones de presentación. Esta interacción es significativa $\left(F^{\prime \prime}(6,588)=2,69 ; p<0,025\right)$ y está ilustrada en la Figura 2. Según nuestras predicciones, a los 6 años y medio las dos condiciones auditivas, $\mathrm{C} 1$ y $\mathrm{C} 2$, son las que permiten mejores puntuaciones, mientras que a partir de los 7 años y medio la condición visual, $\mathrm{C} 4$, es la más favorable.

Centrándonos ahora en la naturaleza de los modelos rítmicos, se comprobó que en las tres edades se daba la misma escala de dificultad. La Figura 3 presenta esta jerarquía de dificultad idéntica en todas las edades para los 4 modelos, y demuestra, por tanto, la falta de interacción entre la edad y los modelos. El modelo M4 es el más difícil de reproducir en todas las edades. Podriamos pensar que ello se debe a tres razones: el elevado número de sus elementos (14), su tempo rápido (120) y las tres duraciones que incluye $(\lambda \downarrow$. $)$. Por el contrario, el modelo $\mathrm{M} 2$ resulta el más fácil en todas las edades: su tempo es rápido (120), pero el número de elementos es reducido (8) y sus duraciones son sencillas ( $\perp$ y $d$ ). Volveremos sobre esta jerarquía en la discusión.

Para terminar este estudio global nos queda revisar la importancia de los ensayos. El cuadro 2 muestra que en el segundo ensayo las puntuaciones son siempre más altas que en el primero $\left(F^{\prime}(1,84)=94,21 ; p<0,001\right.$ de forma global, y $\mathrm{F}=2,72$ a los 6 años y medio; $\mathrm{F}=3,38$ a los 8 años $\mathrm{y}$ medio; $\mathrm{p}<0,01$ en los 3 casos). No se da interacción entre la edad y los 
FIGURA 3

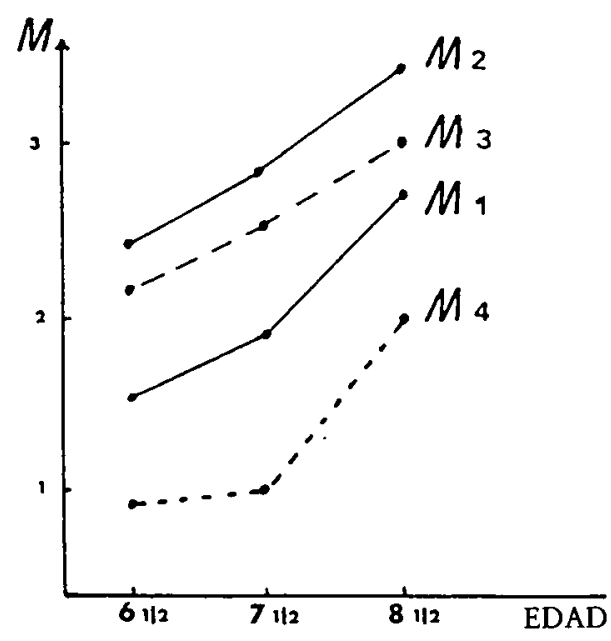

Puntuación media ( $m$ ) obtenida en cada modelo M1 a M4 en función de la edad.

TABLA II

Mejora de las escalas en función de los ensayos

\begin{tabular}{|c|c|c|c|c|}
\hline Edad & $61 / 2$ & $71 / 2$ & $81 / 2$ & $\mathbf{m}$ \\
\hline $\begin{array}{l}\text { Ensayo } 1 \\
\text { Ensayo } 2\end{array}$ & $\begin{array}{l}1,52 \\
2,00\end{array}$ & $\begin{array}{l}1,62 \\
2,44\end{array}$ & $\begin{array}{l}2,55 \\
3,06\end{array}$ & $\begin{array}{l}1,90 \\
2,50\end{array}$ \\
\hline
\end{tabular}

ensayos, ya que los progresos debidos a la repetición son los mismos en cada edad.

\section{Estudio de los modelos de respuesta}

Se puede obtener una misma puntuación total, 1, 2 ó 3, de distintas formas. El análisis de los modelos de respuesta permite concretar qué aspectos del ritmo son tratados de forma adecuada por el niño. Recordemos que estos modelos codifican en la primera columna la presencia de una forma estructurada, en la 2." la sucesión de los intervalos del modelo, en la $3 .^{2}$ la presencia de acentos y en la $4 .^{2}$ columna el respeto del tempo. Hemos utilizado para tratar los datos un programa informático de tri multicriterios elaborado por Gilles Vallée, en el que el usuario concreta el grupo de edad, el modelo, la condición o el ensayo sobre el que se debe efectuar el tri, y el programa calcula las frecuencias de aparición de cada tipo de modelo. La Tabla III presenta, en porcentajes, el reparto por edades de los diferentes modelos.

Con las notas extremas ( 0 y 4 ) sólo es posible un tipo de configuración: una ojeada a la primera y última línea del cuadro nos hace ver que las respuestas sin estructurar son más numerosas $(30,47 \%)$ a los 6 años y medio 
que las buenas reproducciones $(23,06 \%)$, también son numerosas a los 7 años y medio $(27 \%$ ) y raras a los 8 y medio $(8,99 \%)$, edad en la que casi la mitad de las puntuaciones $(42,27 \%$ ) llegan al máximo. En las tres edades casi la mitad de las reproducciones obtienen puntuaciones intermedias $(1,2$ y 3$)$ en las que los diferentes modelos posibles no están repartidos de forma equiparable. La reproducción de una forma bien estructurada, pero sin relación con el modelo, sin acento y con un tempo diferente, es la respuesta más frecuente para la puntuación 1 (modelo 1000). Este tipo de «invención rítmica» apenas varía su frecuencia con la edad, pasando de un $20 \%$ a un 14 aproximadamente. Hemos observado, en otros experimentos, que los niños frecuentemente producen organizaciones típicas (que hemos calificado como "estereotipos") sin tener en cuenta los modelos (Gerard, 1988). En la nota 2, que en conjunto es poco frecuente, sólo un modelo se da con una frecuencia que merece atención, y sólo en niños de 8 años y medio: el que muestra una estructura (aunque «original») y un tempo semejante al del modelo. Este modelo, 1011, designa de nuevo una «invención" rítmica, pero muestra la integración, en esta invención, de la velocidad del modelo y la presencia del acento. A los 8 años y medio no es raro este tipo de respuesta $(15,62 \%)$, y si se suma, en los niños de esta edad, este tipo de respuesta con la anterior (modelo 1011) se comprueba que más de una cuarta parte de las reproducciones $(15,62+9,76=25,38 \%)$ corresponde a una invención, pero adaptada al tempo y a la organización intensiva. La misma suma a los 6 años y medio

\section{TABLA III}

Porcentajes de aparición de cada uno de los catorce modelos posibles en función de la edad. Se han clasificado 256 reproducciones de cada edad. Se han dejado al margen los modelos que superan un $10 \%$ de media en las 3 edades

\begin{tabular}{|c|c|c|c|c|c|}
\hline \multirow{2}{*}{ Nota global } & \multirow{2}{*}{ Modelo } & \multicolumn{3}{|c|}{ Edad } & \multirow{2}{*}{$\mathbf{m}$} \\
\hline & & $61 / 2$ & $71 / 2$ & $81 / 2$ & \\
\hline 0 & 0000 & $30,47 \%$ & $28,13 \%$ & $8,99 \%$ & 22,53 \\
\hline 1 & $\begin{array}{l}0001 \\
0010 \\
0100 \\
1000\end{array}$ & $\begin{array}{r}1,95 \\
0,39 \\
0,39 \\
19,92\end{array}$ & $\begin{array}{c}0,78 \\
0, \\
0, \\
16,01\end{array}$ & $\begin{array}{c}0, \\
0, \\
0, \\
14,45\end{array}$ & 16,80 \\
\hline 2 & $\begin{array}{l}0011 \\
1001 \\
1010 \\
1100\end{array}$ & $\begin{array}{l}1,17 \\
4,30 \\
4,69 \\
0,\end{array}$ & $\begin{array}{l}0,78 \\
1,96 \\
3,12 \\
0,39\end{array}$ & $\begin{array}{l}0, \\
9,76 \\
1,17 \\
0,\end{array}$ & \\
\hline 3 & $\begin{array}{l}0111 \\
1011 \\
1110 \\
1101\end{array}$ & $\begin{array}{l}2,34 \\
8,20 \\
3,12 \\
0,\end{array}$ & $\begin{array}{r}0, \\
11,72 \\
8,59 \\
0,78\end{array}$ & $\begin{array}{c}0, \\
15,62 \\
2,74 \\
0,\end{array}$ & 11,85 \\
\hline \multirow[t]{2}{*}{4} & 1111 & 23,06 & 27,74 & 42,27 & 32,68 \\
\hline & Total & $100 \%$ & $100 \%$ & $100 \%$ & \\
\hline
\end{tabular}


y los 7 y medio da un total bajo (inferior a un $15 \%$ ). Me atrevería a decir, por ello, que los estereotipos sólo se hacen menos estereotipados a los 8 años y medio.

Nos queda revisar la importancia de las condiciones de presentación. El análisis de los modelos de respuesta también permite estudiar cada índice por separado, sin preocuparse de los otros. Si, por ejemplo, de momento sólo nos interesa el tempo, los modelos XXX1 que corresponden a una determinada forma, buena o mala, conforme o no al del modelo, con o sin acento, pero cuyo tempo está cercano al modelo, quedarán retenidos. La Tabla IV presenta, para cada edad y separando las dos condiciones auditivas y las dos visuales, los 3 índices de respuesta siguientes: en relación con la sucesión exacta de intervalos cortos y largos de los modelos (X1XX), en relación con los acentos (XX1X) y en relación con el tempo (XXX1).

¿Se dará la influencia específica de las condiciones auditivas y visuales en todos los índices de puntuación?, ésta es la primera cuestión que vamos a analizar. El cuadro 4 muestra que es así. A los 6 años y medio los niños obtuvieron sistemáticamente mejores puntuaciones en las condiciones auditivas: en cuanto a intervalos conformes al modelo se dio un $34,37 \%$ de reproducciones correctas (contra un $22,66 \%$ en la condición visual), en cuanto a los acentos un $46,09 \%$ contra un $39,06 \%$, para el tempo un $43,75 \%$ contra un $37,50 \%$. La jerarquía de las condiciones era inversa a los 7 años y medio: todos los «mayores" se beneficiaron de las condiciones visuales en cada uno de los tres índices de respuesta examinados. Este hecho vino a reforzar nuestras anteriores conclusiones.

\section{TABLA IV}

Evolución con la edad (en \%) de la reproducción de cada indice de puntuación en. función de la forma de presentación del ritmo. Se registraron 128 reproducciones para cada edad y forma de presentación *

\begin{tabular}{|c|c|c|c|c|c|}
\hline Indices & Modelos & Edad & $\begin{array}{c}\text { Condiciones } \\
\text { auditivas } \\
\text { C1 C2 } \\
(\%)\end{array}$ & $\begin{array}{c}\text { Condiciones } \\
\text { visuales } \\
\text { C3 C4 } \\
(\%)\end{array}$ & $\underset{(\%)}{\mathbf{m}}$ \\
\hline Intervalos & $x 1 x x$ & $\begin{array}{ll}6 & 1 / 2 \\
7 & 1 / 2 \\
8 & 1 / 2\end{array}$ & $\begin{array}{l}34,37 \\
32,03 \\
47,66\end{array}$ & $\begin{array}{l}22,65 \\
42,97 \\
52,34\end{array}$ & $\begin{array}{l}28,91 \\
37,50 \\
50,00\end{array}$ \\
\hline Acentos & $\mathrm{xx} 1 \mathrm{x}$ & $\begin{array}{ll}6 & 1 / 2 \\
7 & 1 / 2 \\
8 & 1 / 2\end{array}$ & $\begin{array}{l}46,09 \\
49,22 \\
64,06\end{array}$ & $\begin{array}{l}39,06 \\
54,69 \\
69,53\end{array}$ & $\begin{array}{l}42,97 \\
51,95 \\
66,80\end{array}$ \\
\hline Tempo & $\mathrm{xxx} 1$ & $\begin{array}{ll}6 & 1 / 2 \\
7 & 1 / 2 \\
8 & 1 / 2\end{array}$ & $\begin{array}{l}43,75 \\
39,84 \\
69,53\end{array}$ & $\begin{array}{l}37,50 \\
47,66 \\
75,78\end{array}$ & $\begin{array}{l}41,01 \\
43,75 \\
72,60\end{array}$ \\
\hline
\end{tabular}

* En cada caso, el porcentaje se estableció sobre 128 observaciones, y no podía pasar de 100. Pero como los tres tipos de modelos que se tuvieron en cuenta no eran excluyentes unos de otros, los totales (por edad) en columna pueden superar el $100 \%$.

La Tabla IV nos permite revisar un último punto. Las medias registradas al margen presentan la evolución con la edad de las tres dimensiones de respuesta, reunidas todas las condiciones. Estas medias muestran que el 
respeto de los intervalos exactos resultó difícil en todas las edades, incluso a los 8 años y medio. Sólo la mitad de las respuestas los respetó a esa edad, pero el progreso entre los 6 años y medio y los 8 y medio fue regular. $\mathrm{Pa}$ rece ocurrir lo mismo con los acentos, pero recordemos que nuestro criterio era muy laxo: bastaba con que se señalara una diferencia de intensidad en cualquier momento de la respuesta para dar la nota 1 . Discutiremos más detenidamente este punto en las conclusiones. Pero, lo que queríamos subrayar fundamentalmente era el «salto» registrado entre los 7 años y medio y los 8 y medio en relación con el tempo. Hasta los 7 años y medio, menos de la mitad de las respuestas mostraban una aproximación correcta a la rapidez de los modelos, mientras que a los 8 años y medio casi lo hacían las tres cuartas partes. La distinción entre modelos lentos (tempo de 60 para M1 y M3) y modelos rápidos (tempo de 120 para M2 y M4) era tardía y se alcanzaba bruscamente.

\section{CONCLUSIONES Y DISCUSION}

En la introducción señalábamos que es la prosodia del lenguaje lo que ofrece un primer marco de organización rítmica a los niños de 4 años. Las onomatopeyas ayudan a los niños más pequeños de entre los que hemos examinado. A la hora de elegir entre las ayudas a ofrecer en el aprendizaje del ritmo, las informaciones auditivas y verbales nos parecen las más apropiadas hasta los 6 años y medio. Otros experimentos también nos han permitido comprobar que niños de 6 años, novatos en cuanto a experiencia musical, mejoraban sus reproducciones de un ritmo si a éste se le añadía una frase significativa, mientras que "músicos" de la misma edad sólo encontraban ayuda si contaban con una melodía (Gerard y Auxiette, 1988). Esto no significa que otras técnicas pedagógicas no tengan fundamento, sino, simplemente, que todo depende de la edad y de los conocimientos anteriores de los niños. A los 7 u 8 años, «espacializar» un ritmo es pertinente, lo cual demuestra que en el niño han aparecido nuevas capacidades que le permiten establecer correspondencias entre informaciones visuales y auditivas. La "espacialización» de un ritmo es una etapa a cubrir en el estudio de la música, ya que la anotación musical se basa también en una analogía entre espacio y duración. Así, en su teoría musical, Chailley y Challan (1947), ilustraban las reglas de escritura musical con la ayuda de la figura que reproducimos a continuación:

FIGURA 4

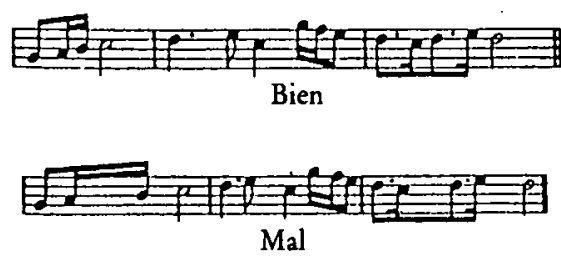

Separación de las notas según su duración. Pensad, cuando anotéis valores diferentes, que un valor largo debe ocupar normalmente más lugar que un valor corto y viceversa. 
Pasemos ahora a la organización rítmica. En nuestra opinión no tiene sentido establecer una constante global de éxito fracaso sobre este aspecto, a no ser que inmediatamente se descomponga en «buenos" y «malos» errores. Un ejemplo de «buen error» sería una combinación estable de dos duraciones en una relación simple $(1 / 2 \circ 1 / 3)$, incluso si esta organización no es la que se pide. Ya hemos visto que estas formas bien organizadas -que hemos llamado invenciones - son muy precoces y frecuentes, pero que la reproducción de la rapidez conforme al modelo es tardía y la reproducción de los acentos es muy rara. Comencemos por el problema de los acentos. De la misma forma que el niño pequeño parece integrar mal informaciones auditivas y visuales, también integra mal las variaciones de intensidad y temporales. Los niños de 6 años integran correctamente secuencias isócronas en las que los acentos aparecen en uno de cada dos golpes o en uno de cada tres, y reproducen correctamente estas secuencias con diferentes velocidades. Pero cuando las secuencias no son isócronas no se produce la acentuación: los niños centran su atención en la organización temporal, aunque se les pida explícitamente que presten atención a los acentos (Gerard y Drake, 1990). Por eso es por lo que nosotros en este experimento nos hemos contentado con un sólo acento situado en cualquier lugar. Pero, incluso así hemos comprobado que a los 6 años y medio menos de una de cada dos reproducciones está acentuada, mientras que a los 7 años y medio lo está una de cada dos; apenas más. Y ello a pesar de que aparecen anacrusas sistemáticas en todos los modelos (salvo en M3), que deberían atraer la atención del niño sobre la aparición tardía del acento, que no se podría confundir con un posible acento subjetivo de comienzo de grupo.

A continuación haremos algunos comentarios sobre la organización temporal. La organización temporal se corresponde mejor con el modelo al aumentar la edad del niño, pero este resultado no es importante. Nos detendremos más en las «invenciones» registradas. $\mathrm{Al}$ analizar los resultados recordábamos los «estereotipos» encontrados en otros experimentos (Gerard, 1988; Gerard y Auxiette, 1989: Drake y Gerard, 1989), combinaciones estables y regulares, muy frecuentes, a menudo repetidas 5 ó 6 veces seguidas por los niños (hasta que se les decía iya es suficiente!) y que tienen de particular el que no corresponden en nada al modelo que debían reproducir. Estos estereotipos comienzan, en general, con un intervalo largo o una serie de 3 ó 4 golpes isócronos, luego se suceden 2 ó 3 intervalos cortos, y un último intervalo largo cierra la figura antes de su repetición. En el presente experimento hay un solo modelo que obliga a la ruptura con este tipo de forma preferencial, el modelo 4 , que exige un agrupamiento de 2 en 2 (corto-largo, corto-largo). Esta puede ser una de las razones que explicaría su mala reproducción. Es indudable que el elevado número de sus elementos sobrecarga la atención y/o la memoria de los niños, pero, recordémoslo, nos bastaba con que los 5 primeros golpes respetaran la sucesión de intervalos cortos y largos de los modelos para dar una puntuación positiva. Puesto que el modelo mejor reproducido (M2) tenía, como el modelo más difícil, un tempo rápido, y puesto que el modelo que quedaba en segunda posición (M3) tenía también tres duraciones diferentes, concluiremos, en términos "gestaltistas", que quizá sea la forma global del modelo M4 la que resulte difícil de tratar por los niños, más que el número de duraciones diferentes o el tempo. 
La imitación del tempo de los modelos, al contrario que las otras dos dimensiones estudiadas, parece tener una evolución irregular, con un salto brusco entre los 7 años y medio y los 8 y medio: sólo los «mayores» parecen prestarle una atención especial, sobre todo en condición visual, en la que el $75,78 \%$ de las respuestas tienen la velocidad exigida. Se eligieron dos tempi: 60 y 120 «a la negra». Una velocidad débil quizá pudiera hacer más difícil la estructuración perceptiva de la secuencia, una velocidad alta pudiera dificultar su realización motora, pero sobre todo las dos velocidades se desvían de lo que se conoce del tiempo motor espontáneo de los niños y del tempo que prefieren oír, en el que los intervalos inter-golpes, son, como media, del orden de medio segundo (en M2 y M4) y de 1 segundo ó 1 y $1 / 2$ (en M1 y M3), nosotros queríamos ver si, con independencia de la precisión de la respuesta, se había integrado el movimiento de conjunto. A los 6 años y medio y 7 y medio los niños estaban en su mayoría en la gama del tempo espontáneo, los niños de 8 años y medio fueron los que presentaron diferencias importantes en el tempo de las reproducciones. La ruptura con las velocidades espontáneas es pues una habilidad perceptivo-motora bien dominada, pero más tardía que la percepción de los acentos o de la sucesión exacta de los intervalos de los modelos.

Nos habría gustado concluir este experimento con algunas precisiones suplementarias en relación con la importancia de las condiciones auditivas. $\mathrm{El}$ «salto» que se da en la condición visual C4 en relación con todas las demás a partir de los 7 años y medio es claro (y estadísticamente significativo), pero no hay ninguna diferencia estadísticamente significativa entre los «titita» de C2 y los «lalala» de C1. No podemos añadir nada más sobre el papel diferenciador de las onomatopeyas de $\mathrm{C} 2$, que sin embargo codifican intervalos cortos y largos de forma específica. No obstante, con una evidente terquedad, hemos vuelto a trabajar sobre estas onomatopeyas en un experimento con niños de 6 años, y esperamos descubrir pronto algo más sobre ellas.

\section{Referencias}

Chailley, J. y Challan, H. (1947). Théorie complète de la musique. París: éditions Musicales.

DAVIDSON, L.; MCKernon, P. y GARDNER, H. (1981). "The acquisition of song: a developmental approach", en Documentary report of the Ann Arbor Symposium, Reston, VA, Music Educators National Conference, 301-315.

DEMANY, L.; MCKENSIE, B. y VURPILLOT, E. (1977). "Rhythm perception in early infancy", Nature, 266, 718-719.

Dowling, W. J. y HARwood, D. L. (1986). Music cognition. Orlando: Academic Press.

DRAKE C. y GERARD, C. (1989). "A psychological pulse train: How young children use this cognitive framework to structure simple rhythms", Psychological Research, 51, 16-22.

FraISSE, P. (1956). Les structures rythmiques. Louvain: Publications Universitaires de Louvain.

- (1974). Psychologie du rythme. París: Presses Universitaires de France.

GERARD, C. (1988a). "L'enfant et le rythme: Etudes expérimentales de la discrimination et de la reproduction de rythmes musicaux", Vibrations, 6, 70-105.

- (1988b). «Perception du temps et du rythme: L'enfant dispose-t-il d'une horloge interne?", Marsyas, 8, 75-85.

- (1989), «Rythmes spontanés et rythmes musicaux chez l'enfant ", Marsyas, 10, 87-94.

GERARD, C. Y AUXIETTE, C. (1988). "The role played by melodic and verbal organisation in the reproduction of rhythmic groups by children", Music Perception, 6, 173-193.

GERARD, C. y DRAKE, C. (1990). "The ability of young children to reproduce intensity differences in musical rhythms?. Perception and Psychophysics, 48 (1), 91-101. 
Hargreaves, D. J. (1986). The developmental psychology of music, Cambridge: Cambridge University Press.

KELLEY, L. y SUTTON-SMITH, B. (1987). *A study of infant musical productivity", en J. C. Peery, I. M. Peery y T. W. Draper (eds.), Music and Child Development, New York: primavera-verano, 35-53.

Martenot, M. (1976). Principes fondamentaux d'éducation musicale et leur application, París: Magnard, 3ème édition augmentée.

- (1981a). Musique en tête. Actes du colloque sur la psychopédagogie de la musique, Issyles-Moulineaux, EAP, coll. Psychologie et pédagogie de la musique, 147-172.

- (1981b). Méthode. Paris: Magnard.

MialareT, J. P. (1979). Pédagogie de la musique et enseignement programmé. Issy-les-Moulineaux, EAP, coll. Psychologie et pédagogie de la musique.

MooG H. (1976). The musical experience of the preschool child, (C. Clarke, trans.), Londres: Schott.

OrfF, C. y KeEtman, G. (1967). Musique pour enfants. Schott Frères.

PETZOLD, R. G. (1981). Auditory perception of musical sounds by the children in the first six grades, Madison, The University of Wisconsin, rapport dactylographié.

SLOBODA, J. A. (1985). The musical mind: the cognitive psychology of music, Oxford, Clarendon Press.

- (1988). Generative processes in music. Oxford: Clarendon Press.

Stambak, M. (1960). "Trois épreuves de rythme», en R. Zazzo (ed.), Manuel pour l'examen psychologique de l'enfant, Neuchâtel: Delachaux et Niestlé.

WASHBURN, K. J. y COHEN, L. B. (1984). Infant perception of rbytbmic form, presented at the International Conference on Infant Studies, New York City, abril 1984, 1-11.

Willems (1983). Panorama pédagogique de l'éducation musicale Willems, stage d'information sur l'éducation musicale, Bayonne.

ZENATTI, A. (1981). L'enfant et son environnement musical, Issy-les-Moulineaux, EAP, coll. Psychologie et Pédagogie de la musique.

\section{El aprendizaje del ritmo musical. C. Gérard. $C L E, 1991,10,85-99$.}

Datos sobre la autora: C. Gérard es psicóloga investigadora en el laboratorio de Psicología Experimental asociado al CNR, equipo de Psico-acústica.

Dirección: CNR, 28 rue de Serpente, 75006 Paris.

Agradecimientos: La autora quiere mostrar su agradecimiento muy especialmente a Christine Rèmond, brillante estudiante de doctorado que ha estado asociada a esta investigación en todas sus etapas, desde la programación del experimento hasta el análisis estadistico, pasando por una larga recogida de datos.

Artículo original: L'Apprentissage du rythme musical: Sur quels indices peuvent s'appuyer les enfants? Les Sciences de l'Education, 1990, 3-4, 63-84. Reproducido con autorización. Traducción de Inés Marichalar.

(C) De todos los artículos. Deberá solicitarse por escrito autorización de CLE para el uso en forma de facsimil, fotocopia o cualquier otro medio de reproducción. CLE se reserva el derecho de interponer las acciones legales necesarias en aquellos casos en que se contravenga la ley de derechos de autor. 